\title{
Crítica de la educación por competencias
}

\section{Angélica del Rey ${ }^{1}$ \\ J. Sanchez-Parga}

Este reciente libro se hace eco de una larga tradición crítica, que desde algo más de una década combate un proyecto educativo tan peligroso como insidioso, ya que ha logrado penetrar el sistema escolar y universitario, por todo el mundo, con el pretexto de renovarlo y mejorarlo. Más que una recensión de esta obra, este texto constituye toda una reflexión de lo que nos merece el problema planteado; sobre todo por la vigencia que sigue cobrando la nueva educación capitalista, y que en las últimas dos décadas ha devaluado casi un 30\% las carreras de profesores de colegio y de Universidad (Laval, 2011; Bouzidi, Jaaidane y Gary-Bobo, 1960-2004). En estos momentos, septiembre de 2011, maestros, profesores y estudiantes protestan en las calles de Chile, de España, de Francia, y no hace mucho habían manifestado de inconformidad también en Inglaterra e Italia. Este "malestar en la educación" responde a múltiples factores y se expresa en morfologías diversas; la educación por competencias podría ser un síntoma de todas ellas.

Antes la educación y formación universitaria se orientaban al desarrollo de las capacidades intelectuales del sujeto, prescindiendo de los posibles campos y de las posibles formas de sus usos, empleos o aplicaciones; hoy la educación y formación universitarias se orientan a desarrollar aquellos conocimientos para ser aplicados a determinadas competencias. Mientras que la educación y formación universitarias dirigidas al desarrollo de las capacidades se construyen basándose en las "libertades" concretas de los individuos, la educación por competencias instrumentaliza los conocimientos y refuerza su utilitarismo (Nussbaum, 2011).

Los ideólogos de las competencias han tratado de incorporar éstas a la educación universitaria, sin una previa reflexión crítica sobre lo que significan las competencias, cuál es su origen y de dónde proceden, cuál es su contexto ideológico y, sobre todo, las incompatibilidades y contradicciones entre el modelo de saberes y destrezas propios de las competencias y el conocimiento científico, teórico y crítico; el que hace pensar y desarrolla el pensamiento, que siempre

1 A l'Ecole des Competences. De l'éducation à la fabrique de l'élève performante (La Découverte, Paris, 2010). 
había sido propio de la educación secundaria y universitaria. Para compensar esta falta de tratamiento crítico de las competencias y su adaptación a los sistemas académico científicos de la Universidad, los ideólogos de las competencias han procedido de manera inversa: adaptar los procesos y procedimientos epistemológicos propios de la docencia universitaria al esquema de las competencias.

Varias son las consecuencias que resultan de tales prácticas, y que pueden observarse en muchos de los estudios y artículos que se han escrito y siguen escribiéndose sobre el tema. En unos casos, los más coherentes o radicales, la aplicación de las competencias a la docencia universitaria devastan la especificidad científica y académica de ésta, cambiando la formación de los futuros profesionales. En otros casos, la introducción de las competencias es casi más nominal o declarativa que efectiva, real en algunos campos más apropiados a este modelo educativo, pero irrelevante en otros ámbitos del saber y la formación universitaria. Aunque como situación intermedia o de transición encontramos propuestas híbridas, que intentan combinar con más o menos coherencia un modelo académico-científico de docencia universitaria con una enseñanza aprendizaje modulada por competencias. En cualquiera de estos casos, lo que aparece claro es que el programa de las competencias ha encontrado mayores resistencias en países donde hay un arraigado pensamiento educativo, mucho más sólido y con mayor tradición, mientras que las competencias arrasan en sistemas educativos e instituciones universitarias más nuevas y sin una fuerte tradición educativa y científica.

\section{La transformación capitalista de los saberes en competencias.}

El gran proyecto de la Unión Europea, fundado en las competencias y competitividades económicas se ha plasmado en el proyecto de la "Universidad empresa", y desde entonces la obsesión por las competencias y su impacto en la escuela y en la formación de los enseñantes no ha dejado de arraigarse y extenderse por los sistemas educativos de todo el mundo (Boutin y Jullien, 2000). Y sin embargo, hay que seguir resistiendo al "asalto neoliberal de la educación", defendiendo el principio de que "la escuela no es una empresa" (Laval, 2003).

Había que capitalizar todos los recursos educativos, economizando gastos (más estudiantes) y rentabilizando costos (menos clases, colegios y enseñan- 
tes); ahora de lo que se trata es de capitalizar los mismos conocimientos, mercantilizarlos bajo la forma de competencias.

Las competencias es otra de las nuevas ideologías, que colonizan los sistemas educativos actuales: un proceso neoliberal tendiente a colocar al estudiante al servicio de las necesidades de la economía y del mercado, y no la educación al servicio del estudiante. Se trata de reducir la educación a la fabricación de un alumno económicamente "performante"; adiestrado para ser competitivo en los mercados profesionales y del trabajo.

Se define la competencia como "un saber hacer fundado sobre la movilización y utilización eficaz de un conjunto de recursos"; "un empleo organizado de un conjunto de capacidades y habilidades, y de conocimientos aplicado en situaciones determinadas" (Lasnier, 2002: 32). Se trata fundamentalmente de una enseñanza/aprendizaje atomizado en una multitud de competencias, modulando así los (des)conocimientos de acuerdo a determinadas demandas o necesidades expresadas desde las empresas o los mercados laborales y profesionales. Lo que impide este modelo educativo es que los conocimientos y competencias enseñados y aprendidos sean comprendidos y explicados, pensados, descontextualizados, no comparados ni relacionados entre sí. Este modelo modular de la educación más bien atrofia el desarrollo de la inteligencia.

La enseñanza por competencias invierte los objetivos de la enseñanza, al supeditarlos a los de la evaluación, convirtiéndose ésta en el criterio principal del aprendizaje, y no éste en criterio de la evaluación: según esto "el estudiante debe ser evaluado para mejor aprender y no aprender para ser evaluado" (Del Rey, 29). Como si lo que el estudiante pueda aprender se redujera a lo que pueda ser evaluado. "Implementar una enseñanza ceñida a las modalidades de la evaluación", significaría que el estudiante solo aprende aquello que puede ser objeto de evaluación (Rope \& Tanguy, 1994: 104). Como si no debiera y pudiera aprender mucho más de lo que puede ser evaluado. El imperativo de la evaluación impone una enseñanza de conocimientos ya organizados y formateados por el criterio de su utilización para ser competentes, pero descuida o relega el aprendizaje de organizar los conocimientos.

Se trata de un modelo educativo fundamentalmente "heteroconstructivista", ya que las enseñanzas dictadas desde un sistema de competencias establecido por criterios de cuestionable validez educativa, resultan exteriores al sistema de 
aprendizaje del niño o del joven. Por otro lado, se pretende compensar la orientación "heteroconstructivista" del conocimiento en la educación del niño y adolescente con declaraciones de un "autoconstructivismo" radical, como si tales declaraciones compensaran la fundamental orientación "heteroconstructivista" de las competencias aprendidas. Nada casual que los ideólogos de las competencias recurren a esa otra moda pedagógica de los "autismos educativos", la "autoeducación", la "autoenseñanza" y el "autoaprendizaje", del estudiante actor educativo y protagonista del aula, y sujeto tan principal como casi exclusivo de su propia formación, relegando al profesor a la condición de "facilitador". Qué lejos quedamos de concebir al profesor, más bien, como un "dificultador", el que descubre las dificultades al alumno, que cuanto más difíciles son los conocimientos que comprende más se desarrolla su inteligencia.

Los ideólogos de las competencias no ignoran que el sujeto del aprendizaje es el estudiante, pero desconocen que la subjetividad de este aprendizaje es la inteligencia de ese mismo estudiante, y que la educación consiste precisamente en el desarrollo de esa inteligencia y de todas sus facultades. Por el contrario, la educación por competencias no se orienta al desarrollo de la inteligencia del estudiante, sino más bien y más directamente a determinados ejercicios y desempeños, usos y funciones de dicha inteligencia. Es aquí donde reside el principal error epistemológico y pedagógico de la educación por competencias. De la misma manera que las competencias se enseñan separadamente, sin pensar las múltiples relaciones entre ellas, las que permitirían pensarlas con una cierta coherencia y racionalidad, así mismo se aprenden separadamente, cuando pretenden legitimarse o enriquecerse pedagógicamente recurriendo a recetas muy atractivas, supuestamente novedosas y de fácil circulación como aquella de "saber, saber-hacer y saber-ser", como si los conocimientos, sus efectos subjetivos y comportamentales pudieran ser separados y modulados tan fácilmente como son catalogados. Conocer, ser y actuar es una misma cosa, siendo en una relación dialéctica de estas tres dimensiones de la sujetividad del conocimiento, que puede construirse un real proceso educativo.

Tal confusión pedagógica y de formación universitaria se explica por dos razones: a) un déficit de pensamiento educativo, incapaz de pensar la pedagogía como una teoría del aprendizaje y una práctica de la enseñanza; este principio epistemológico de la pedagogía garantiza los fundamentos autoconstructivistas 
del aprendizaje y la complementariedad heteroconstructivista de la enseñanza; b) pensar la educación y formación universitarias no desde los conocimientos y desde los sujetos de dicha educación y formación, sino desde las ofertas y demandas de los conocimientos enseñados y aprendidos, desde los posibles mercados laborales y profesionales, donde se aplicarán tales competencias.

Es obvio que los criterios de formación profesional y de "empleabilidad" futura de los estudiantes no pueden estar ausentes de la educación universitaria, pero más que supeditar ésta a aquellos, hay que pensar aquellos criterios de profesionalización como en parte implicados y en parte consecutivos de los principios y orientaciones fundamentales de la educación. De igual manera, se cree que la educación por competencias prepara y adapta los estudiantes a una sociedad competitiva y a la concurrencia que enfrentará en el futuro, cuando en realidad ocurre todo lo contrario: con la enseñanza por competencias el estudiante aprende la competitividad, y se prepara a construir y contribuir a un mundo de rivalidades.

\section{Las competencias contra los saberes}

Son muchos los autores que ven en estos nuevos modelos educativos "el eclipse del saber" y el proceso "hacia una sociedad de la ignorancia". Tras el imperceptible cambio de orientación (pues ya no se trata de adaptar los públicos a los saberes sino éstos a los públicos), se gesta el proyecto neoliberal de "reconstruir los diferentes ámbitos de la vida social según el modelo del mercado económico y en especial los dos espacios que más resistirían a sus pretensiones: la política y el conocimiento" (Gauchet, 2009: 164).

Lejos de orientarse al desarrollo de la inteligencia del estudiante y de la racionalidad de sus facultades, la educación por competencias responde más bien a exigencias de facilidad y utilidad y, en definitiva, a demandas de mercados: "la finalidad de este aprendizaje no es más que la normalización disciplinaria, adaptada al utilitarismo neoliberal" (Del Rey, 113). La lista de competencias

2 Lindays Waters, L'Eclipse du savoir, Allia, Paris, 2008; Marcel Gauchet, "Vers une société de l'ignorance?", Le Débat, n. 156, sept. - oct. 2009. 
se establece de manera exterior al alumno, que debe adquirirlas, al margen del desarrollo de su inteligencia, al margen de la estructura y fases del proceso de aprendizaje: "las competencias presuponen al fin de cuentas un hombre sin cualidades, superficie lisa capaz de adquirir las "buenas" competencias independientemente de las cualidades que dispone y las que dispone la situación a la cual está confrontado" (ibíd.: 120).

Las competencias suponen un modelo de aprendizaje por elecciones (pick up), como si fueran artículos en la estantería de un supermercado, sin relación racional entre ellas, y por agregaciones sin una articulación subjetiva que las organice y les confiera coherencia intelectual. Esto hace que la "adquisición por competencias" sea opuesta al real proceso de aprendizaje, donde la secuencia de conocimientos se articulan racionalmente entre sí, "al desconocer el proceso de emergencia de lo útil sobre el fondo de lo inútil; de otro lado, el sujeto aprende de hecho articulando sus comportamientos propios a la situación concreta, materialmente determinada, a la cual se encuentra confrontado" (íbíd.: 122).

La educación por competencias elimina la "eficacia paradójica" esencial al proceso educativo, que consiste en ese aprendizaje por parte del estudiante, que va más allá de toda la enseñanza, el que es un proceso de socialización, cuya importancia transciende los procesos de saber y saber-hacer intelectuales (cfr. Prost, 1930; Perrin, 1981). Es ilusorio creer que la educación por competencias termina por "personalizar" éstas en el estudiante, y que pueden llegar a incorporarse como parte de su inteligencia. Lo que ocurre es todo lo contrario: el aprendizaje por competencias termina "formateando" la inteligencia del estudiante, atrofiando su desarrollo y creatividad. Es obvio que un modelo de aprendizaje que no activa las sinapsis del cerebro, lo que hace inteligente a un niño y adolescente, incurre en una suerte de desecerebralización de los estudiantes.

El desplazamiento de la finalidad educativa corresponde a una "laboratorización de la formación", que suplanta cualquier nueva teoría del aprendizaje por una demanda social de eficacia del sistema educativo, "conduciendo a reformular las exigencias de los empleadores en función de principios de políticas de gestión de mano de obra y de profesionales baratos, y traducidos en 
objetivos de aprendizaje. ${ }^{3}$ Se considera que las competencias están dotadas de un valor intelectual autónomo, cuando en realidad se encuentran estrechamente dependientes de la adaptación a un tipo de sociedad y de demandas que las valorizan en una óptica económica. La validez y efectividad intelectuales de las competencias son tan efímeras y contingentes como las demandas a las que responden y los contextos de su aplicación (cfr. Bottani \& Vrignaud, 2005: 120).

Mientras que la inteligencia y sus facultades no pueden medirse, las competencias no es más que aquello que puede ser medido por un test $u$ otro instrumento; más aún las competencias son definidas, enseñadas y aprendidas sólo para ser evaluadas y medidas. Mientras que cada inteligencia releva de una fundamental subjetividad, y en cierto modo es única, la educación y evaluación de las competencias presupone la uniformidad del aprendizaje, favorece al nivel global la generalización a todos los sistemas educativos del mundo, permitiendo que: a) todos los estudiantes y profesionales puedan circular como mercancías por todos los mercados laborales y del empleo; b) que todos ellos puedan competir entre sí en función de sus competencias.

Bajo este capital común de conocimientos y competencias, existen las competencias claves, con mayor o menor rendimiento y rentabilidad; y en el mercado de las competencias están las estrategias de desarrollo de "recursos humanos", y la ideología del "capital humano" y su gestión empresarial. Por eso, la idea de competencias no se reduce a la relación educativa y al modelo de enseñanza/aprendizaje, sino que se sitúa la noción de competencia en el centro de un nuevo modelo institucional, al permitir articular por medio de la educación la valoración del capital propio de cada individuo, la rentabilidad de la inversión educativa y sus apuestas del crecimiento económico.

De la misma manera que bajo la fase industrial y productiva del desarrollo capitalista la "fuerza de trabajo" podía ser vendida por el obrero como si fuera una mercancía, y el capital podía medirla y calcularla y, sobre todo, pagarla de acuerdo a sus rendimientos, así también en la moderna sociedad del conocimiento las competencias tienen la extraordinaria ventaja de convertirse en una

3 Cfr. Sylvie Montchetren, L' “aproche par competences", technologie de rationalisation pédagogique, <www.cereq.fr, net.dec.36>. 
mercancía inmaterial, pero que puede ser calculada, medida y evaluada, vendida y comprada en los mercados laborales y profesionales.

A diferencia de los títulos y diplomas, que calificaban personalmente al estudiante y su historia educativa, las competencias están fundadas por "perfiles" y "experticias", y serán objeto de certificados. Los títulos y diplomas educativos eran conferidos por el Estado y significaban un reconocimiento social y público; por el contrario, las competencias son certificadas por las empresas y los mercados: "la educación fundada sobre competencias implica el desplazamiento del papel legitimador del Estado a favor del Mercado, nuevo encargado de valorizar y de legitimar los conocimientos convertidos en competencias evaluables por la empresa". Cada vez son más las empresas, como CISCO (ejemplo de industria de redes informáticas en el mundo entero), que forman a sus profesionales durante uno, dos o tres años, y certifican sus competencias en el empleo de tales tecnologías por un determinado período de años, al cabo de los cuales los certificados pierden su valor. Las competencias son hoy el caballo de Troya de las empresas en la Universidad.

\section{Una formación para lumpen profesionales}

El mercado neoliberal necesita, por un lado, de profesionales cada vez más y mejor preparados, especialistas, grandes científicos y pensadores, capaces de producir nuevas ideas y nuevas tecnologías, que generen y entiendan los cambios sociales, orientando su desarrollo, y que en definitiva integren la clase dirigente; pero por otro lado, también necesita una clase mayoritaria de intelectuales "proletas" o "precarios", lumpen profesionales únicamente formados para algunas competencias, muy baratos y para empleos provisionales y bajos salarios, solo útiles para ciertas actividades instrumentales, para determinadas fases de la producción y de la aplicación tecnológica, de las más simples y elementales. Se trata en definitiva de trabajadores y empleados poco reciclables, muy desechables y fácilmente sustituibles por una nueva generación con el mismo perfil de lumpen profesional.

El filósofo francés Jean Claude Michea llama al "aprendizaje por competencias" La enseñanza de la ignorancia, la cual consiste en aprender destrezas y habilidades, prácticas técnicas y aplicaciones de saberes, pero sin necesidad 
de aprender y comprender esos saberes, conocimientos y tecnología (Michea, 2006). Para las competencias solo se aprenden aquellos saberes para ser utilizados y aplicados, pero no para ser aprendidos y explicados, y sobre todo para poder generar nuevos u otros saberes. Los profesionales de y para las competencias son simplemente subsidiarios de las máquinas y de los aparatos, de las organizaciones e instituciones, de los procesos sociales.

El aprendizaje de competencias consiste en un aprendizaje de la ignorancia, porque supone aprender conocimientos que otros han pensado, pero que no se entienden ni se comprenden; solo se aplican. Se trata de ignorar lo que se aprende o aprender lo que no se entiende y tampoco se puede explicar. Para lo que no prepara la enseñanza/aprendizaje por competencias es a pensar, a producir conocimientos y crear tecnologías.

El aprendizaje por competencias es el resultado terminal y más perverso de la masificación universitaria: el programa consiste en introducir en la misma Universidad la gran desigualdad de clases existente en la sociedad: entre aquellos que piensan y generan pensamiento y aquellos que únicamente consumen las ideas de los otros, y solo son diestros en competencias y en servir a los otros. La educación por competencias prepara un vivero de mano de obra barata, flexible, para responder a mercados laborales de mano de obra poco calificada: "se trata de garantizar un crecimiento significativo de empleos para trabajadores del sector de servicios en la venta al pormenor y la distribución, así como para otras ocupaciones elementales, que no requieren cualificación formal o muy poca". Hasta ahora la Universidad y el conocimiento habían valido para reducir las desigualdades y garantizar iguales oportunidades con el fin de lograr una mayor igualdad social; hoy el mercado y la dominación neoliberal se sirven del aprendizaje por competencias para consolidar las desigualdades sociales junto con las desigualdades del conocimiento ya desde la misma Universidad.

Mientras que la sociedad industrial del siglo XIX produjo el lumpen proletariado, la mano de obra precaria y desechable, la moderna sociedad del conocimiento del siglo XXI produce su propio lumpen estudiantado y profesional, a

4 Centro Europeo para el Desarrollo de la Formación Profesional (Cedefop), "Future skill needs in Europe: medium-term forecast. Background technical report", Publications Office of the European Union. Luxembourg, 2009: 
través entre otras procedimientos del aprendizaje por competencias. Este es la razón no solo teórica, científica y académica, sino especialmente ética y política para oponerse al aprendizaje por competencias.

Colegas ingenuos o presas propicias de las modas educativas, movidos sin duda por las mejores intenciones, pero sin una suficiente alerta crítica, están dispuestos a aceptar como lo mejor el último slogan pedagógico, la última receta o consigna educativa, el último modelo de docencia o enseñanza aprendizaje; y todo ello sin interrogarse de donde proceden tales innovaciones, y creyendo que los cambios se justifican por sí mismos. Únicamente porque es nuevo se lo considera mejor, sin preguntarse de donde viene la innovación, cuál es su ideología implícita, quien la fabricó y la subvencionó, quien se encarga de difundirla, a qué intereses responde y en definitiva qué efectos y consecuencias puede tener. Esto mismo sucedió ayer con la "lucha contra la pobreza", el "crecimiento económico con equidad", la "gobernabilidad" primero y la "gobernanza" después, y lo mismo ocurre con el "aprendizaje por competencias".

Dos cuestionamientos de fondo objetan los presupuestos educativos y de profesionalización de la "formación por competencias".

En primer lugar, el problema de la educación o formación por competencias es que sus mismos presupuestos, los más implícitos, no la justifican. Lo que parece fundamentar y orientar las competencias es su mejor y más adaptado nivel de profesionalización de los estudiantes y su mayor capacidad para responder a las demandas de los mercados laborales y profesionales. Ahora bien, es la misma estructura y funcionamiento de dichos mercados, y en particular sus cambios cada vez más rápidos, los que a mediano e incluso a corto plazo invalidan las competencias adquiridas en tiempos cada vez más breves. Las competencias que hoy adquiere el estudiante en la Universidad pueden ya no ser requeridas en un par de años, cuando las demandas profesionales y laborales, tecnológicas y de know how sean otras y diferentes. En este sentido la formación por competencias prepara profesionales efímeros, fácilmente desechables y difícilmente reciclables; pues solo aprendieron determinadas competencias pero no saben aprender otras.

De otro lado, los mercados laborales y profesionales generan demandas cada vez más específicas y especializadas, nichos de competencias muy precisos y estrechos, de tal manera que no solo es muy difícil lograr elevados niveles 
de especialización y competir por ellos, sino que además tales especializaciones "de punta" hacen todavía más arduo que competencias tan especializadas puedan reconvertirse y reciclarse con otras. A ello hay que añadir la creciente movilidad de las tecnologías y de las mismas ofertas laborales y profesionales como también de las demandas. Consecuencia de este fenómeno es la tendencia constatada desde hace un par de décadas del creciente porcentaje de universitarios que desempeñan profesiones o encuentran empleos en campos diferentes, en ocasiones muy diferentes, a los de sus carreras o estudios universitarios. La razón es obvia desde el punto de vista de las demandas y de los mismos mercados laborales/profesionales: en sus procesos de innovación, de cambios y de especialización, son muchas veces los universitarios de otras carreras y disciplinas o áreas del conocimiento, los que más pueden aportar en términos de interdisciplinariedad, innovación y especialización. Pero la razón es también obvia desde los mismos universitarios, que con una formación suficientemente sólida, amplia y profunda, son capaces de desempeñarse en futuros campos profesionales y laborales tan cambiantes como diversificados y diferentes de aquellos que fueron sus ámbitos de estudio.

Por todas estas razones es un brutal engaño lo que sostiene la OCDE: "los empleadores han reconocido en ellas (las competencias) factores claves de dinamismo y de flexibilidad. Una fuerza de trabajo dotada de estas competencias es capaz de adaptarse constantemente a la nueva demanda y a medios de producción en continua evolución" (Pont \& Werquin, 2001).

Dinamismo empresarial para sustituir mano de obra desgastada e incapaz de reciclarse para las nuevas competencias requeridas por los cambios tecnológicos y medios de producción; proceso todo él regido por una tendencial reducción de los salarios: "para un nivel de demanda dado, correspondiente a un cierto tipo de competencias, el aumento de la oferta resultará en una reducción de los salarios reales para todos los trabajadores, que disponen ya de estas competencias". ${ }^{5}$ Son los mismos textos oficiales que asimilan las competencias a la doble precarización laboral y salarial.

5 "Progress towards the Lisbon objectives in education and training", documento de trabajo del personal de la Comisión, 2005, ref: SEC (2005) 419. Cfr. Nico Hirt, "En Europe, les compétences contre le savoir", en Le Monde Diplomatique, n. 679, oct. 2010. 
En segundo lugar, el otro problema es de orden epistemológico, y tiene que ver con la especificidad de la educación o docencia universitaria. Cuando hace algo más de una década se pone de manifiesto la crisis de la educación universitaria, se atribuye esta crisis a dos problemas, que nada tienen que ver con la especificidad de dicha educación/formación universitaria: se pensó en una problemática pedagógica, de enseñanza/aprendizaje de los conocimientos, y a un problema de comunicación entre docentes y estudiantes. Tales planteamientos del problema eran falsos y no hicieron más que encubrir y agravar la cuestión de fondo: se pedagogizó todavía más la docencia universitaria, despojándola de su cientificidad, y se confundió la comunicación científica con un problema comunicacional y no científico. No es recurriendo a las pedagogías y didácticas y mucho menos a las nuevas TICs (tecnologías de la información y comunicación) que se resuelve la crisis de la docencia y formación universitarias sino recuperando y fortaleciendo su especificidad científica. ${ }^{6}$

En la Universidad los conocimientos no son enseñados para que sean aprendidos, sino que son explicados para ser comprendidos, y para que a su vez los mismos estudiantes sean también capaces de explicarlos en la medida que los han comprendido. En la Universidad se enseña y se aprende no conocimientos sino como pensarlos y como producirlos. Ya que en la Universidad no es cualquier tipo de conocimientos que son enseñados y aprendidos, sino los conocimientos científicos; es decir aquellos que son comprendidos y explicados, y que por consiguiente son pensados y desarrollan una inteligencia crítica; la que garantiza no solo el nivel de cientificidad, de certeza o de verdad de tales conocimientos sino también la capacidad de juzgar críticamente otros conocimientos. Y en este sentido, en la educación universitaria el problema de la docencia no es de comunicación entre profesores y estudiantes, sino un problema de comunicación de ambos con los conocimientos científicos.

Es este modelo de Universidad, este modelo de docencia y formación universitarias, este modelo de conocimientos y de relación con los conocimientos, que las competencias tienden a liquidar.

6 Para un desarrollo más amplio de esta problemática cfr. J. Sánchez Parga, La docencia universitaria. Para un manifiesto antipedagógico, Universidad Politécnica Salesiana, Abyayala, Quito, 2003. 


\section{Bibliografía}

\section{Textos}

Bottani, Norberto \& Pierre Vrignaud. La France et les évaluations internationales. Paris: La Documentation Française. 2005.

Boutin, Gerald y Louise Jullien. L'obsession des competences. Son impact sur l'école et la formation des enseignants. Montréal: Editions nouvelles. 2000.

Centro Europero para el Desarrollo de la Formación Profesional (Cedefop), "Future skill needs in Europe: medium-term forecast. Background technical report", Publications Office of the European Union. Luxembourg, 2009:

Laval, Christian. L'Ecole n'est pas une entreprise. Le néoliberalisme à l'assaut de l'enseignement publique. Paris: La Découverte. 2003.

Lasnier, François. Réussir la formation par competences, Montreal: Edit. Guérin, 2002.

Michea, Jean Claude. L'enseignement de l'ignorance et ses conditions modernes, Paris: Climats. 2006.

Prost, Antoine. Historie de l'enseignement et de l'éducation IV, déspuis 1930, Paris: Perrin. 1981.

Rope, François \& Lucie Tanguy (dir.), Savoirs et competences. De l'usage de ces notions dans l'école et dans l'entreprise. Paris: L'Harmattan. 1994.

Pont, Beatriz \& Patrick Werquin, "Nouvelles competences: vraiment?" en: L'Observateur de l'OCDE, Paris: avril, 2001.

"Progress towards the Lisbon objectives in education and training", documento de trabajo del personal de la Comisión, 2005, ref: SEC (2005) 419. Cfr. Nico Hirt, "En Europe, les compétences contre le savoir" en: Le Monde Diplomatique, n. 679, oct. 2010.

Sánchez-Parga, José. La docencia universitaria. Para un manifiesto antipedagógico,

Universidad Politécnica Salesiana, Quito: Ed. Abya-Yala, 2003.

Waters, Lindays. L'Eclipse du savoir. Paris: Allia. 2008. 
Gauchet, Marcel. "Vers une société de l'ignorance?" Le Débat, n. 156, sept. oct. 2009.

\section{Sitios web}

Montchetren, Sylvie. L' “aproche par competences", technologie de rationalisation pédagogique, $<$ www.cereq.fr, net.dec.36>. 